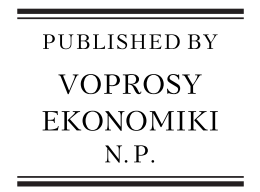

\title{
Long-term development of the global energy sector under the influence of energy policies and technological progress
}

\author{
Alexey A. Makarov ${ }^{a}$, Tatyana A. Mitrova ${ }^{\mathrm{b}}$, \\ Vyatcheslav A. Kulagin a,* \\ ${ }^{a}$ Energy Research Institute of the Russian Academy of Sciences, Moscow, Russia \\ ${ }^{\mathrm{b}}$ Energy Centre of the Moscow School of Management SKOLKOVO, Moscow, Russia
}

\begin{abstract}
The world energy sector is entering a period of transformation, defined by the emergence of a whole range of cost-effective technologies and the formation of new state priorities that can radically change the structure of energy use. The calculations of the development of world energy markets for the period up to 2040 were made using economic and mathematical optimization modeling based on the ERI RAS SCANER model complex, for two scenarios: Conservative (maintaining key benchmarks and regulatory measures within the framework of existing energy policies, as well as incremental development of technologies without major breakthroughs) and Energy Transition (assuming ambitious goals in the field of reducing emissions, as well as accelerated technology development). The results of calculations show that the growth rate of primary energy consumption will slow down significantly. Renewable energy sources (RES) will be able to provide up to $35-50 \%$ of electricity production and up to a quarter of the world's energy consumption by 2040 , while the share of fossil fuels will decline. Oil and coal will pass the peak of consumption before 2040. This will not only lead to a radical change in the price environment of energy markets, but also to a transformation of the way they are organized and regulated, as well as to a revision of business models of most energy companies.
\end{abstract}

Keywords: world energy, energy development, energy prices, oil, gas, coal, electricity. JEL classification: Q41, Q42, Q43, Q47.

\section{Introduction}

State energy policies can have a significant impact on the competitiveness of energy sources, on priorities for technological development, on trade flows and

\footnotetext{
* Corresponding author, E-mail address: vakulagin@yandex.ru
} 
on demand in end-use sectors. They become a determining factor in the transformation of energy balances of countries and world regions. The World Energy Council (WEC) has formulated three main goals that energy policy in each country is focused on in one way or another, the so-called Energy Trilemma: the availability of energy, its environmental impact and reliability of supply (WEC, 2019).

The current stage of global energy development is characterized by the expansion of energy supply options due to the development of trade and the emergence of a whole range of new technologies in the production, transportation and consumption of energy. As a result, the availability of energy and opportunities to improve the reliability of energy supply increase. If previously many countries had virtually no choice of sources of energy supply, and the most accessible source was used, nowadays a wide array of options is becoming available, suited for varying demands.

Against the background of growing global concerns about climate change and environmental pollution, the relevance of environmental issues has increased significantly. The global environmental agenda is largely based on decisions taken within the framework of the UNFCCC. One of the most significant events of the UNFCCC conferences was the signing of the Kyoto Protocol in 1997, aimed at creating a global international emission trading system, but its implementation hindered at regional level. The failure to reach an agreement on the second commitment period under the Kyoto Protocol has created uncertainty about further action. It was only at a conference in Paris in 2015 that a new agreement was reached. In part, it was a step back, because instead of global market mechanisms for managing emissions in the framework of Kyoto, it was decided to limit to the agreement to non-binding national obligations, which the countries themselves formulated. But in the absence of consent on other formats, this agreement was a certain breakthrough.

After 2015, under pressure from public opinion and growing concern about climate issues, many countries have moved ahead of the curve and taken more decisive action on climate initiatives. Tightening environmental policies led to the spread of emission taxes and emission trading systems, as well as physical restrictions on the use of particular fuels, and the development of government incentives for the introduction of alternative energy sources with a wide array of support mechanisms. As a result, the development of renewable energy is accelerating and its share in the energy balance is expanding, and fossil fuel technologies solutions leading to minimizing emissions are becoming increasingly relevant. But the transformation of energy is not limited to changing the structure of the energy balance - there is also a qualitative change in energy systems.

\section{Economics and technology in the context of the energy trilemma}

The parameters of the "Energy Trilemma" differ from region to region and from country to country. For some, the environmental concerns are the top priority. Typically, these are countries with high per capita income that can afford to invest in more than just basic necessities. But for the poorest countries, the environment is often the last priority. This does not mean that low-emission energy 
sources are not relevant in these countries. It is possible, for example, to actively use solar panels near the equator in countries with low per capita incomes, but the key issue will not be the environmental policies, but their ability to provide electricity at lower prices compared to fossil fuels.

Recently, significant progress has been made in reducing the cost of certain types of renewable energy sources (RES), particularly wind and solar energy. At the same time the R\&D in the field of fossil energy has also not been idle - thanks to the new technologies, significant volumes of previously inaccessible reserves have become available for development as well as cost-competitive. Reliability and costs in pipeline transportation have undergone significant improvement; the overall efficiency of fuel combustion has increased.

In the power and heat generation sector, the innovations in the field of production based on fossil and renewable sources, the introduction of digital and intelligent systems, and the development of technologies for energy storage and transmission have been the highlights. Increasing efficiency of power and heat production based on fossil sources is achieved mainly by:

- applications of combined power and heat generation (cogeneration);

- transition to steam turbine installations with supercritical steam parameters, installation of boilers with a circulating fluidized bed in coal generation;

- use of cutting-edge steam-gas and gas-turbine equipment in gas generation.

All of the manufactured equipment under various operating schemes is constantly being improved in terms of increasing efficiency, improving operating modes, and meeting stricter environmental requirements. Environmental standards and the introduction of carbon taxes and emissions' trading systems have stimulated the development of low-emission combustion chambers, filters, soot traps, membrane technologies for air and exhaust separation, technologies for transporting and storage of carbon dioxide and flue gases, and other combustion products.

In the nuclear industry the main areas of development are: the fast neutron reactors; the new generation of light water reactors; closed fuel cycle technologies in reactors on fast and thermal neutrons; the development of breeder technology. In the long term, high hopes are pinned on controlled thermonuclear fusion.

In renewable energy, the main technological progress areas are:

- reducing the cost of solar energy production through the use of non-silicon and cascade photo converters;

- reducing the cost of production of wind energy by reducing the cost of construction and equipment that is not related to the turbine;

- improving the efficiency and reliability of large-scale hydropower plants and solutions for small hydroelectric plants;

- improving the parameters of geothermal installations, including the binary cycle;

- working out new solutions in the field of heat and electricity production using biofuels and waste.

The largest increase in renewable energy capacity is expected through the use of solar and wind installations. In the period 2010-2018, the cost of solar-based electricity generation was reduced by 1.5-4 times (depending on the type of technology and country), offshore wind power - by about $15 \%$, and onshore wind installations - by 30\% (IRENE, 2019). These advances can be attributed to three reasons: improved technologies, economies of scale, 
and production localization in countries with low costs, especially in China. According to ERI RAS estimates, the cost of solar power may be further reduced by up to $50 \%$ by 2040 . For wind power the prospects for costs reduction are more modest - up to $35 \%$ for offshore and up to $20 \%$ on land. This is because wind power technologies and techniques have already been perfected; therefore the main source of cost reduction is optimization of subsidiaries and reducing the production costs.

The development of digital and intelligent technologies, including smart grids, microgrids, virtual power plants, and the Internet of things, will significantly change the power system. All this combined will gradually make the system more flexible and distributed. Smart controllers on the consumption side will be able not only to automatically manage modes based on the system load and the required power, but also act as a supplier of electricity to the network, if they possess the capacity.

The rapid development of renewable energy sources (RES) poses new challenges for energy systems related to the divergence of peaks in electricity production and consumption. This has significantly increased the role of fossil fuel generation as a balancing element. Smart systems on the consumer side can partially solve the problem of unevenness by distributing the load, where possible, over non-peak periods. But the key will be progress in the field of energy storage. However, these accumulators will have to be more cost-effective than alternative solutions (for example, by using UGS and gas generation), and not exceed the cost difference of electricity in different periods of time. The success in scaling up and reducing the cost of energy storage will determine the systematic restrictions for the use of RES (Makarov et al., 2017). It is obvious that in the next 15 years, fossil fuels will remain the main source of power balancing for RES, and the extent to which this task can be taken over by storage systems in the future depends in no small part on costs' dynamics.

Nowadays, the largest storage capacities in the world are the hydro-accumulating systems for industrial power storage. Thermal storage systems are second, followed by other mechanical and chemical systems. So far, the economics of storage based on chemical sources (lithium, sodium-sulfur, lead, flow, etc.) are inferior to traditional HPP. But the high demand for new solutions, supported by large-scale investment in $\mathrm{R} \& \mathrm{D}$, in the coming 20 years may lead to a reduction in the cost of storage by 1.5-3 times from the current \$100-700/MWh. Another storage method is the conversion of electricity to hydrogen, but at this stage the costs of this method are significantly higher than the others presented. At the same time, hydrogen (in combination with natural gas) is also considered by fossil fuels producers as one of the options for switching to green energy.

Technologies in the consumer sectors are developing in a wide range of areas, which is caused both by the aim to reduce the cost of energy used, and to improve the consumer characteristics of equipment, devices, infrastructure, etc. (Fig. 1). Among the key consequences of these changes are a steady increase in energy efficiency, the emergence of multiple options in many segments in terms of the energy source used, and the creation of conditions for the transformation of many consumers in the electrical system from passive to active participants due to the possibility of decentralized production and storage. One of the most obvious examples of multi-options is the increasing competition in the transport 

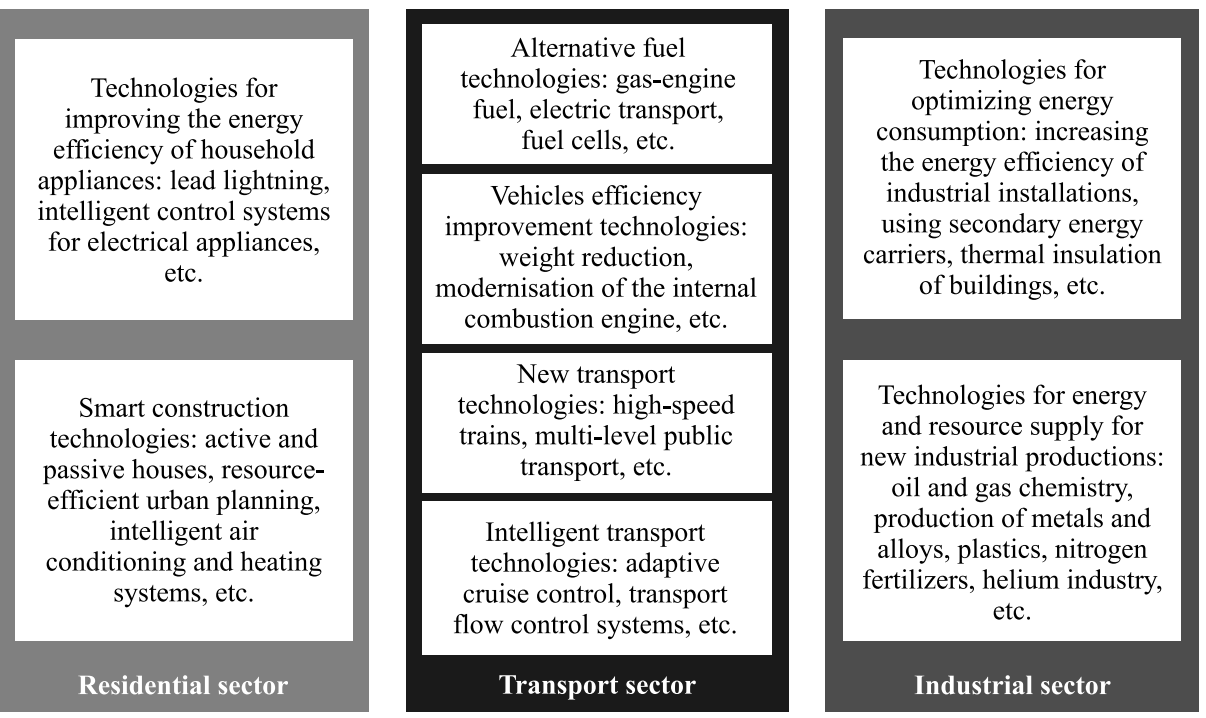

Fig. 1. Key areas of technology development in energy consumption sectors.

Source: Makarov et al. (2016).

sector as technology advances in electric vehicles. But in many cases this is possible thanks to the extensive regulatory support.

\section{The speed of innovation implementation}

Oftentimes, new technologies are perceived as world-changing. Indeed, the advent of affordable computers, mobile phones, and the Internet has radically altered the way of life for most people in a relatively short period of time. But the actual impact of any new technology is very much dependent on the dynamics of their spread, determined by development and manufacturing time, as well as infrastructural and consumer turnover rate. For consumer electronics, computers and smartphones, ten years is a sufficient period to transition of more than $50 \%$ of the devices to the next generation. But when talking about the housing sector, urban infrastructure, nuclear power plants, hydroelectric power plants, aviation equipment, the cycle of technology introduction and market spread may easily exceed 50 years (Fig. 2). Accordingly, the replacement of energy resources in the case of switching to alternative technologies will occur at different rates depending on the consumption segment.

\section{The changes of energy balances}

Based on ERI RAS modelling complex SCANER (Makarov and Filippov, 2011) by means of economic and mathematical optimization modeling, calculations of the development of world energy markets for the period up to 2040 were conducted. Two scenarios were assumed. The first involves maintaining key benchmarks and regulatory measures in the framework of existing energy policies, as well as the incremental development of energy technologies without major breakthroughs. The second scenario involves more ambitious solutions in 


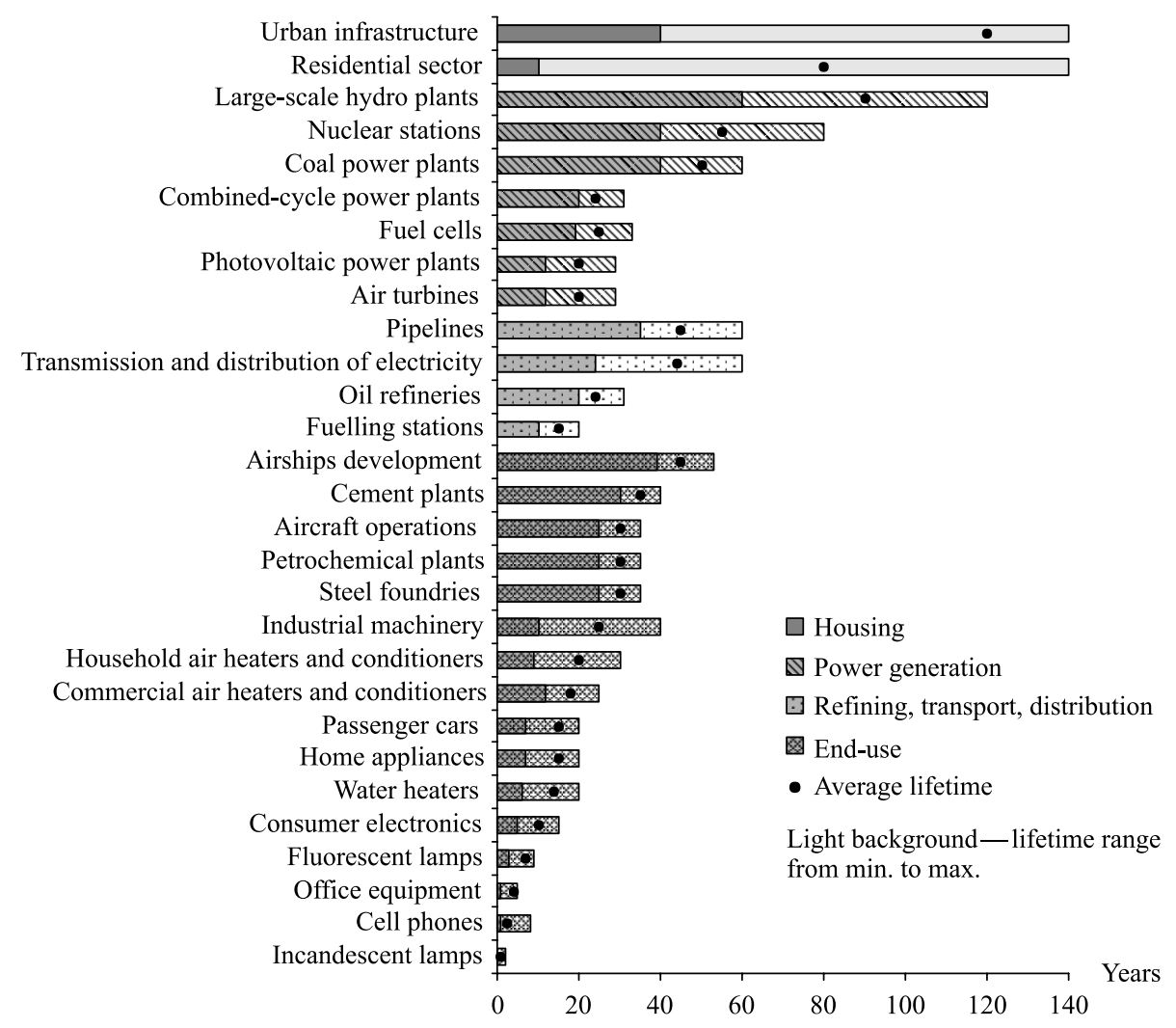

Fig. 2. Infrastructure and technology turnover period.

Source: Kulagin (2020).

the field of reducing emissions, as well as accelerated development of technologies in several areas, in particular in the field of energy storage. The economic and demographic indicators for both scenarios are the same - the average annual global GDP growth rate in $2015-2040$ is $2.8 \%$, and the population is assumed to grow to 9.2 billion people by 2040 .

As the results of calculations demonstrate, fossil fuels will continue to dominate the energy balance until 2040. Yet their share will decrease from $81 \%$ in 2015 to $69-75 \%$ by 2040 . The share of oil and coal will decrease the most, while natural gas will be able to actually increase its contribution to the energy balance.

In the electric power industry, there is a trend for reduction of the dominance of fossil fuels in the next 20 years. In the Energy Transition scenario, only $43 \%$ of electricity will be produced from oil, gas and coal, but if conservative trends in energy development are maintained, they will provide $55 \%$ of production, compared to $66 \%$ in 2015 (Fig. 3). Thus, the direction of the expected changes becomes obvious, but their pace may be different depending on the conditions of global energy development.

\section{Competition development}

Increasingly often, consumers find themselves able to switch from a single energy supply option to a whole range of alternative solutions. In these condi- 
(a) Energy consumption structure

2015

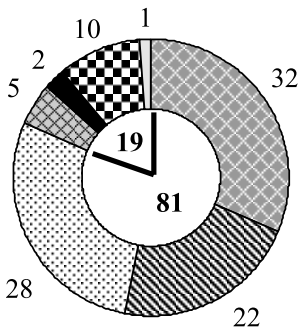

22

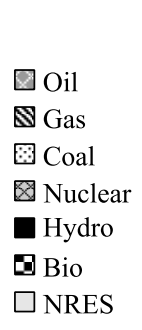

2040 Conservative

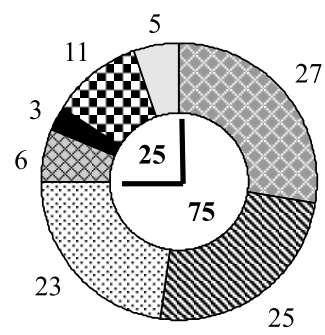

25
2040 Energy Transition

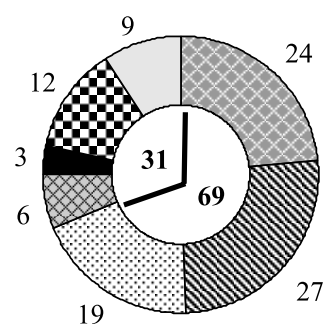

(b) Power generation structure

2015

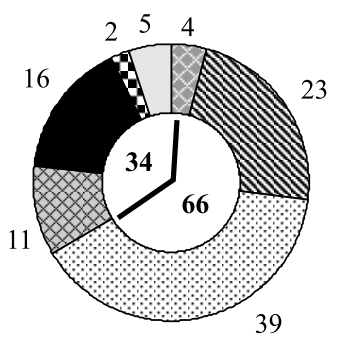

2040 Conservative

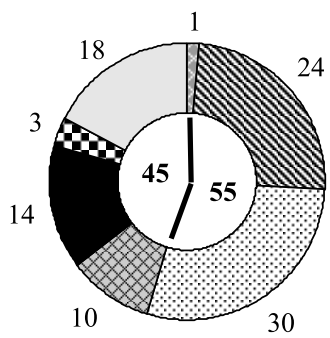

2040 Energy Transition

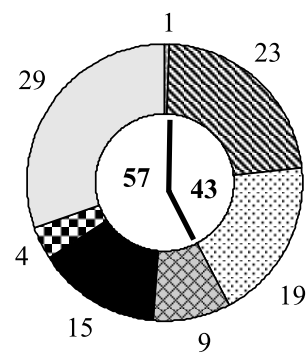

Fig. 3. Structure of global energy consumption and power consumption in 2015 and 2040 by scenario (\%).

Note: NRES - New renewable energy sources.

Source: Authors' calculations.

tions, there is a movement from supplier competition within a single fuel market to multi-vector technological competition. If recently many companies were working in the total absence of alternative to their products, or competing with 1-5 participants in their own market, then in the future they will face dozens of possible solutions offered by companies working in various energy industries.

If costs and prices increase in one of the fuel markets, the market will be ready to offer a whole range of alternatives in the medium term, which sets quite strict cost restrictions, within which companies will have to operate. Already, there are actually a number of technologies in the queue that are waiting for market niches to open due to changes in prices, imposed policy restrictions, or as part of their own development and cost reduction.

\section{Prices and pricing}

In the mid-20th century, oil competed with other fuels in the electric power industry and in fact it was the only liquid energy market-gas supplies were dominated by pipelines that strictly link the producers and consumers, and the coal market had a regional character, mainly with long-term supplies between specific contractors. As a result, oil prices have become benchmarks for other fuels. Over time, the market moved to the second stage, when the share of oil in electricity generation decreased, but by convention, other fuels were still either 
1st stage: Oil prices reflect the market

- Inter-fuel competition in the electric power industry and the flexibility of the oil market allowed it to become a determinant for the prices of other fuels

2nd stage: Oil prices are the benchmark

- Due to established conventions and in the absence of liquidity in the markets of other fuels, oil prices remain the benchmark for all fossil fuels

3rd stage: Establishment of the independent competition-based fuel markets

- Each fuel market generates its own price indicators

4th stage: Price integration through interfuel competition

- The price boundaries of fuel markets are being blurred, and the world is moving to real inter-fuel competition

Fig. 4. The stages of pricing interrelations in the fuel markets.

Source: Compiled by the authors.

directly linked to its prices, or were guided by the dynamics of the oil market during trading. At the same time there was a fairly clear correlation between oil prices and the prices of other fuels (Fig. 4). The almost complete withdrawal of petroleum products from the power industry and the emergence of opportunities to build competitive markets led to the transition to stage 3 , in which prices for coal and gas were mainly formed within the framework of their own short-term liquid trade. In the future, the transformation of pricing will continue. Increasing interfuel competition in several consumption sectors at once will lead to a struggle between niches for fuels, which will directly affect prices and their correlation. As a result, prices will again become largely interconnected, but the number of factors affecting them will increase significantly. And one of these factors will be the cost of emissions.

The calculations show that the world will not face previously announced peaks in oil, gas and coal production due to the depletion of reserves. The approach of peaks is already visible, but for a completely different reason - due to the limits of demand. Accordingly, there is no reason for an increase in energy prices. On the contrary, there are significant restrictions on fossil fuels price levels associated with inter-fuel competition, when the shortages in one fuel are readily compensated by other sources.

Shock of the pandemic and the recession in the global economy will be changing the range of possible conditions of the markets. In the medium-term, until 2025 , the prices dynamics in the oil market will largely depend on the presence of agreements between producers to regulate market balance. If such agreements are in place, the prices can stay above the $\$ 50 / \mathrm{bbl}$ level. If there is no coordination, the excess supply can push the price below $\$ 20 /$ bbl (Fig. 5). In the long term, prices will primarily depend on the dynamics of demand and the corresponding capacities on the supply side.

The situation for the gas market is more stable. In all scenarios, demand for gas is set to grow in the next 20 years. But this does not mean a return of gas prices to levels above $\$ 450 / \mathrm{tcm}$ in Europe and $\$ 600 / \mathrm{tcm}$ in Asia. The world will inevitably have to switch to more expensive production projects, but in parallel, 


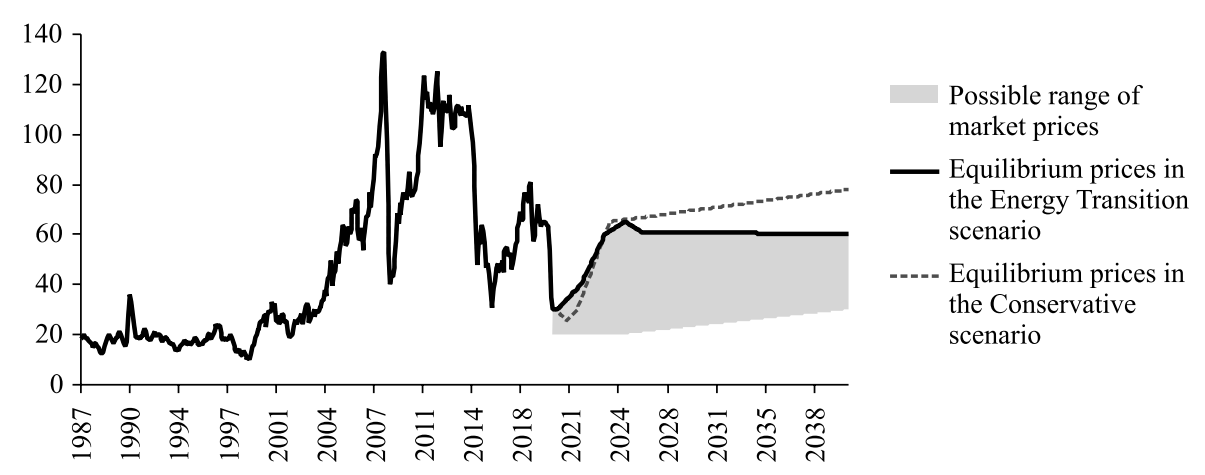

Fig. 5. Oil prices in the Conservative and Energy Transition scenarios (2019 U.S. dollars per barrel).

Source: Authors' calculations.

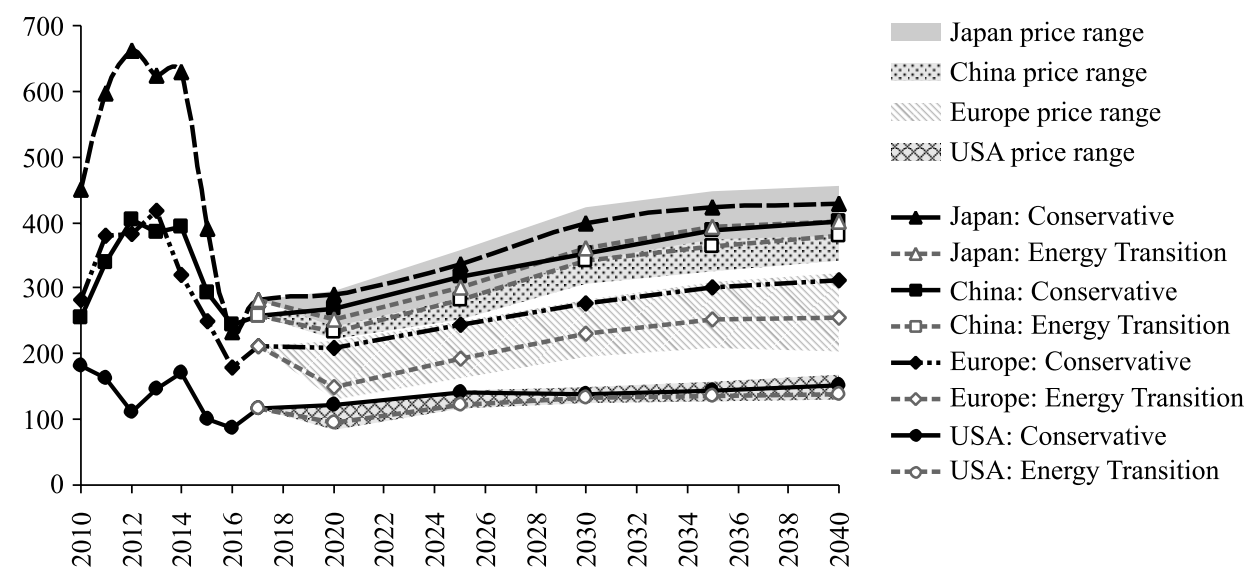

Fig. 6. Gas prices in Conservative and Energy Transition scenarios (2019 U.S. dollars per 1000 cubic meters).

Source: Authors' calculations.

the increased competition will force businesses to optimize costs for all closing projects, and government authorities will have to ease the tax burden. In this situation, prices should be expected to recover to the level of breakeven cost with subsequent stabilization. At the same time, an excess of supply, especially in the period up to 2025 , can lead to market prices by $20-25 \%$ below equilibrium (Fig. 6).

Coal prices in scenarios of an accelerated coal phasing out will be under pressure from oversupply due to the excessive production capacities. Environmental regulation, including $\mathrm{CO}_{2}$ emissions taxes, will exert an additional downward pressure. As a result, coal prices trend at relatively low levels in the conservative scenario and a drop significantly in the Energy Transition scenario (Fig. 7).

The development of renewable energy will have quite a pronounced impact on price dynamics. For countries with cold climates, solar energy will increase the seasonal imbalance in demand for fossil fuels, and thus result in greater seasonal price differentiation. In short time periods, imbalances in the production of solar and wind energy can lead to both drops in prices even to negative levels and 


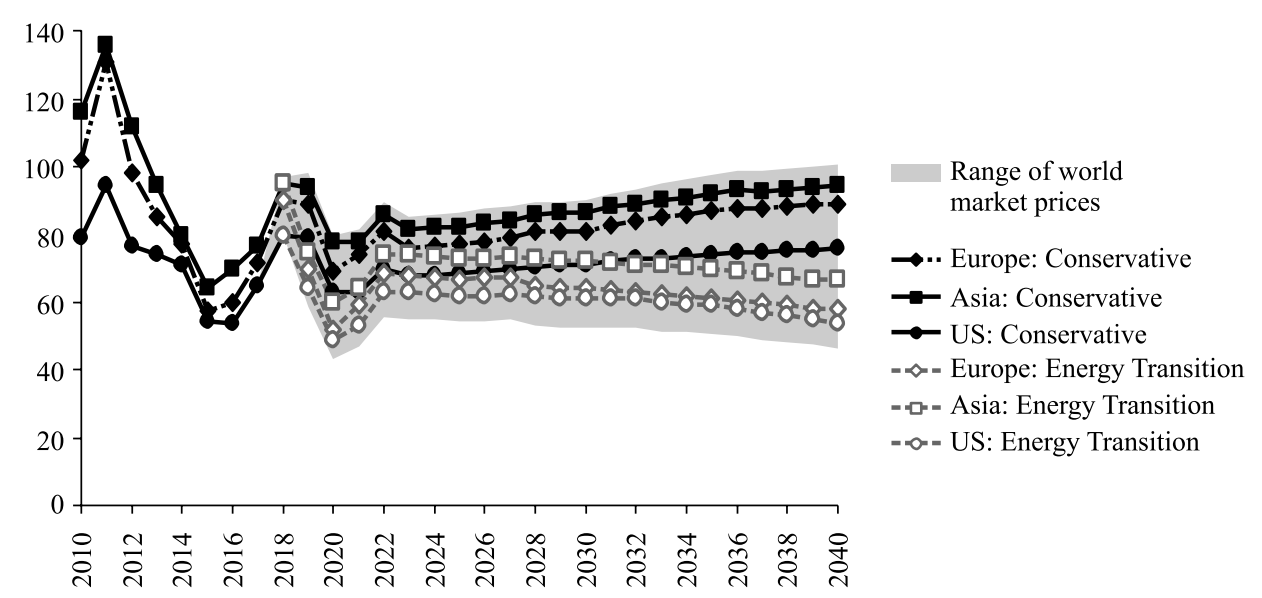

Fig. 7. Coal prices in Conservative and Energy Transition scenarios (2019 U.S. dollars per tonne).

Source: Authors' calculations.

to their growth by several times. This will also affect the dynamics of fossil fuel prices. In addition, changing the structure of the power industry will inevitably condition the need to develop new regulatory rules to ensure the long-term sustainability of these markets.

Prices of fossil fuels for end-users will be significantly higher than wholesale prices in many countries, due to tax and customs regulations. This regulation will be based not only on the need for budgets' revenues, but also on the promotion of energy conservation and support for alternative energy sources in order to ensure their competitiveness.

\section{Conclusion}

The global energy industry has entered the next stage of transformation. The key results of this process will be:

- slowing down the growth rate of primary energy consumption;

- passing the peak consumption of coal and petroleum products;

- increasing the share of renewable energy to $35-50 \%$ in electricity production and up to a quarter of global energy consumption;

- restraining the growth of energy prices through a wide range of market alternatives.

One of the key features of the future energy sector will be high technology competition, both within each fuel market and at the inter-fuel level. Consumers will have multiple options when choosing their preferred energy supply solution, and manufacturers will have a fairly wide choice of business configurations. At the same time, energy companies will have to face the need for continuous technological improvement and development of new business models in the competition for consumers to secure their place in the market. The pandemic and the recession of 2020 will have a significant impact on energy markets in the long-run. Restoration of the growth and development of the global economy will be affected by the energy transition. 


\section{References}

WEC (2019). World energy trilemma index 2019. World Energy Council.

IRENA (2019). Renewable power generation costs in 2018. Renewable Energy Agency.

Kulagin, V. A. (Ed.) (2020). Prospects for global energy development under the influence of technological progress. Moscow: ERI RAS.

Makarov, A., \& Filippov, S. (2011). SCANER modelling and information complex. Moscow: ERI RAS.

Makarov, A. A., Grigoryev, L. M., \& Mitrova, T. A. (Eds.) (2016). Global and Russian energy outlook 2016. Moscow: ERI RAS and ACRF.

Makarov, A. A., Mitrova, T. A., Veselov, F. V., Galkina, A. A., \& Kulagin, V. A. (2017). Perspectives of the electric power industry amid the transforming global power generation markets. Thermal Engineering, 64(10), 703-714. https://doi.org/10.1134/S0040601517100081 